\title{
CIFOPLASTIA NO TRATAMENTO DA FRATURA VERTEBRAL POR INSUFICIÊNCIA - AVALIAÇÃO FUNCIONAL PROSPECTIVA
}

\author{
KYPHOPLASTY IN THE TREATMENT OF VERTEBRAL COMPRESSION \\ FRACTURE - PROSPECTIVE FUNCTIONAL EVALUATION \\ CIFOPLASTIA EN EL TRATAMIENTO DE LA FRACTURA VERTEBRAL POR INSUFICIENCIA - \\ EVALUACIÓN FUNCIONAL PROSPECTIVA
}

\author{
Bartolomeu Ribeiro Coutinho Filho ${ }^{1}$, Thiago Miller Santana Silva ${ }^{1}$, Carlos Eduardo Gonçales Barsotti ${ }^{1}$, Francisco Prado Eugênio dos Santos²,
} Jefferson Alves Galves², Carlos Eduardo Algavez Soares de Oliveira ${ }^{3}$

\begin{abstract}
RESUMO
Objetivo: Avaliar o resultado funcional e o grau de satisfação do tratamento cirúrgico utilizando cifoplastia em pacientes com fratura vertebral por insuficiência localizada na coluna tóraco-lombar. Métodos: Foi realizado um estudo prospectivo em pacientes adultos, com diagnóstico de fratura vertebral por insuficiência com evolução superior a oito semanas, apresentando dor no local da fratura e edema ósseo evidente ao exame de RM. A avaliação funcional foi realizada através do Oswestry Disability Index 2.0 e da Escala Visual Analógica de Dor no pré- e pós-operatório. A satisfação pessoal com o tratamento foi quantificada pela escala de Johnson. Resultados: Houve melhora significativa da dor com uma queda média de 6.4 pontos na Escala Visual Analógica de Dor ao final de doze meses de seguimento em comparação ao período pré-operatório ( $p<00,5)$. A avaliação funcional mostrou $88 \%$ de resultados excelentes ou bons, sendo que sete pacientes (41\%) apresentaram excelentes resultados e oito pacientes (47\%) tiveram resultados bons. Dois pacientes (12\%) mantiveram sua avaliação funcional inalterada. Quanto à graduação subjetiva de satisfação, 82\% declararam-se satisfeitos sendo que 59\% estavam completamente satisfeitos e 23\% satisfeitos com mínimas restrições. Três pacientes (18\%) declararam-se insatisfeitos com o resultado do procedimento. Conclusão: A cifoplastia mostrou-se efetiva em melhorar a função e liberar a dor em pacientes com fratura vertebral por insuficiência. A ocorrência de uma complicação grave (IAM) chama atenção para a necessidade de suporte hospitalar adequado durante a realização do procedimento.
\end{abstract}

Descritores: Fraturas da coluna vertebral/cirurgia; Osteoporose/complicações; Fraturas por compressão/cirurgia.

\begin{abstract}
Objective: To evaluate the functional outcome and degree of satisfaction of surgical treatment using kyphoplasty in patients with vertebral compression fractures located in the thoracolumbar spine. Methods: Prospective study in which adult patients diagnosed with vertebral compression fracture with a course over eight weeks, with pain at the fracture site and bone edema evident by MRI imaging were identified and evaluated for the study. The functional evaluation was performed through the Oswestry Disability Index 2.0 and Visual Analogue Scale of Pain pre- and postoperatively. The personal satisfaction with treatment was measured by the scale of Johnson. Results: A significant improvement in pain with an average fall of 6.4 points in the Visual Analogue Scale of Pain at the end of twelve months of follow-up compared to preoperatively $(p<00.5)$. The functional evaluation showed $88 \%$ excellent or good results, with seven patients $(41 \%)$ with excellent results and eight patients (47\%) with good results. Two patients (12\%) maintained their functional assessment unchanged. As to the subjective degree of satisfaction, $82 \%$ said they were satisfied, and of those, $59 \%$ were completely satisfied and $23 \%$ satisfied with minimal restrictions. Three patients (18\%) reported being dissatisfied with the outcome of the procedure. Conclusion: Kyphoplasty has proven effective in improving the function and relieving the pain in patients with vertebral compression fracture. The occurrence of a serious complication (AMI) calls attention to the need for proper hospital care during the procedure.
\end{abstract}

Keywords: Spinal fracture/surgery; Osteoporosis/complications; Fractures, compression/surgery.

\section{RESUMEN}

Objetivo: Evaluar el resultado funcional y el grado de satisfacción del tratamiento quirúrgico mediante cifoplastia en pacientes con fractura vertebral debido a la insuficiencia situada en la columna toracolumbar. Métodos: Se realizó un estudio prospectivo en pacientes adultos, con diagnóstico de fractura vertebral por insuficiencia, con tiempo de evolución de más de ocho semanas, con dolor en el sitio de la fractura y edema óseo evidente por resonancia magnética. La evaluación funcional se realizó mediante el Índice de Incapacidad de Oswestry 2.0 y la Escala Analógica Visual del Dolor antes y después de la operación. La satisfacción personal con el tratamiento fue medida por la escala de Johnson. Resultados: Una mejora significativa en el dolor con una caída promedio de 6,4 puntos en la escala visual analógica del dolor al final de doce meses de seguimiento, en comparación con el período preoperatorio ( $p<0,05)$. La evaluación funcional mostró 88\% de resultados excelentes o buenos, con 07 pacientes (41\%) que tuvieron resultados excelentes y 08 pacientes (47\%) buenos resultados. Dos pacientes (12\%) se mantuvieron sin cambios la evaluación funcional. En cuanto al grado subjetivo de satisfacción, $82 \%$ dijeron que estaban satisfechos y 59\% se mostraron satisfechos por completo, y el 23\% satisfechos con restricciones mínimas. Tres pacientes (18\%) informaron estar insatisfechos con el resultado del procedimiento. Conclusión: La cifoplastia ha demostrado ser eficaz en la mejora de la función y la liberación del dolor en pacientes con fractura vertebral por insuficiencia. La aparición de una complicación grave (Infarto Agudo del Miocardio llama la atención sobre la necesidad de atención hospitalaria adecuada durante el procedimiento.

Descriptores: Fracturas de la columna vertebral/cirugía; Osteoporosis/complicaciones; Fracturas por compresión/cirugía.

1. Medico Estagiário de Cirurgia da Coluna (R5) do Hospital do Servidor Publico Estadual - São Paulo (SP), Brasil.

2. Medico Assistente de Cirurgia da Coluna do Hospital do Servidor Publico Estadual - São Paulo (SP), Brasil.

3. Mestre em Ortopedia do Hospital do Servidor Público Estadual - São Paulo (SP), Brasil.

Trabalho realizado no Hospital do Servidor Publico Estadual (HSPE) - São Paulo.SP. Brasil.

Correspondência: Rua Borges Lagoa, 1755, Sala 180, Vila Clementino. São Paulo SP. Brasil. CEP.: 04038-034. Email: bartolomeucoutinho@uol.com.br

Recebido em 30/03/2011, aceito em 27/07/2011. 


\section{INTRODUÇÃO}

A Osteoporose é uma doença osteometabólica caracterizada pela perda de massa óssea e a ocorrência de fraturas por fragilidade ${ }^{1}$. Fraturas vertebrais por insuficiência podem resultar em dor, perda de altura (causada pelo colapso vertebral), instabilidade vertebral e deformidade cifótica ${ }^{2}$

De acordo com a classificação de diagnóstico da OMS, a osteoporose é definida através da densidade mineral óssea (DMO) no quadril ou na coluna vertebral quando inferior ou igual a 2,5 desvios padrão abaixo da população de referência ${ }^{1}$. A osteoporose é a doença óssea mais comum nos seres humanos, e representa um importante problema de saúde pública. Estima-se uma ocorrência 1.5 milhões de fraturas osteoporóticas/ano nos EUA, sendo que 700.000 ocorrem na coluna vertebral. A mortalidade em 2 anos para pacientes com fraturas da coluna por osteoporose é 1,5 vezes maior que a do grupo controle de pacientes na mesma faixa etária e é igual à mortalidade dos pacientes com fratura do fêmur proximal, sendo que a maioria dos pacientes com fratura por osteoporose da coluna vertebral apresenta comorbidades associadas ${ }^{3-6}$.

Dor crônica e deformidade cifótica levam a depressão, redução do apetite, deterioração da função pulmonar, perda da capacidade ambulatória e queda na qualidade de vida, resultando em morbidade significante e morte ${ }^{3-7}$. Assim, há crescente interesse no desenvolvimento de métodos de estabilização dessas fraturas que reduzam ou eliminem a dor, permitindo retorno as atividades habituais em curto período de tempo.

O uso de instrumentação segmentar com parafusos de pedículo através de uma abordagem posterior tradicional tem apresentado alta taxa de falha em ossos osteoporóticos ${ }^{8-11}$; consequentemente, técnicas de instrumentação anterior mais enxerto ósseo estruturado, cages e placas foram introduzidos para aumentar a estabilidade da coluna anterior e mostraram-se efetivas ${ }^{12-16}$. Entretanto, estabilização anterior requer uma abordagem mais invasiva que é associada um tempo cirúrgico e de internação hospitalar prolongados, perda sanguínea, complicações do sítio doador de enxerto e, aumento da morbidade e mortalidade em pessoas idosas ${ }^{12-15}$.

O presente estudo procurou avaliar prospectivamente a eficácia da cifoplastia em melhorar a função e liberar a dor em pacientes com fratura vertebral por insuficiência localizadas na coluna tóraco-lombar.

\section{MÉTODOS}

Entre janeiro de 2009 e fevereiro de 2010 dezessete pacientes com diagnóstico de fratura vertebral por insuficiência com tempo de evolução superior a oito semanas e ainda sintomáticos (apresentando dor no local da fratura) e edema ósseo ao exame de ressonância magnética foram identificados e avaliados para participar do estudo. Como critérios de exclusão consideramos: presença de doença metastática na coluna vertebral e presença de déficit neurológico. Os pacientes elegíveis para o estudo assinaram termo de consentimento livre e esclarecido aprovado pelo comitê de ética do Hospital do Servidor Público Estadual de São Paulo.

A técnica cirúrgica foi realizada sob anestesia geral através de uma incisão de $0,5 \mathrm{~cm}$ feita lateralmente aos pedículos da vértebra acometida. Um fio guia de $11 \mathrm{G}$ (Jamshidi needle, Bone access 11-gauge Kyphon, Sunnyvale, CA) é inserido até a borda superolateral do pedículo na incidência AP (2 horas na posição do relógio para direita e 10 horas para a esquerda). No perfil o guia é progredido até atingir a cortical posterior do corpo, verificando sempre se o fio não penetra na parede medial do pedículo no AP. Um guia de $2 \mathrm{~mm}$ é passado pelo fio, que é trocado por uma cânula de trabalho (Osteointroducer, Kyphon). Essa cânula é avançada pela parede posterior da vértebra. A biopsia óssea é obtida inserindo a cânula enquanto se aplica sucção com a seringa. Uma broca manual é inserida para criar o pertuito dentro da vértebra para a passagem do cateter do balão. Esse procedimento é repetido no lado contra lateral.

O balão (Kyphon) é inserido. O balão tem $1,5 \mathrm{~cm}$ e suporta $4 \mathrm{ml}$ de fluido. Cada balão tem 2 marcadores radiopacos, que têm que passar além da ponta da cânula de trabalho para assegurar o enchimento do balão. Os balões são inflados sob intensificação de imagem continua. Os critérios para parar de inflar os balões são: redução da fratura, balão violou algum limite ósseo, pressão maior que 300 psi, ou quando o balão atingir seu volume máximo. A pressão pode ser lida no manômetro do material e o volume na seringa. $\mathrm{O}$ cimento é preparado com 40 cc de Simplex P (Howmedica, Mahwahh, NJ), e $6 \mathrm{~g}$ de sulfato de bário, além de $10 \mathrm{ml}$ de manômero líquido. Já dentro dos tubos de 1,5 ml, aguardamos o cimento ficar pastoso. Em seguida, ele atinge uma consistência em que ao ser empurrado para fora do tubo o cimento enrola-se em torno do mesmo. Os balões são esvaziados e removidos e o cimento com a consistência adequada é colocado na cavidade através de tubos de preenchimentos especiais (Bone void filter tubes, Kyphon) e empurrados com as mãos vagarosamente. O volume de balão inflado e a aparência radiografica durante o preenchimento são os guias da quantidade de cimento a ser colocado. $O$ volume total a ser colocado varia de 1 a $5 \mathrm{ml}$ e é repetido do outro lado.

Para avaliação dos resultados foram utilizados a Escala Visual Analógica (EVA), avaliação funcional Oswestry Disabity Index 2.0 (ODI) e a Graduação Subjetiva de Satisfação de Johnson e cols. A Escala Visual Analógica de Dor é graduada de 0 a 10 onde o menor valor equivale a ausência de dor e o maior valor equivale a dor de mais forte intensidade. Na avaliação funcional através do Oswestry Disabity Index 2.0 (ODI), o paciente emiti uma pontuação referente a uma série de atividades do cotidiano como presença de dor, cuidados pessoais, capacidade de elevar peso e sono. No pré-operatório os resultados são estratificados em incapacidade mínima; incapacidade moderada; incapacidade intensa; aleijado e inválido. No pós-operatório aplica-se o mesmo questionário, mas os resultados são estratificados em excelente; bom; inalterado e piora. A Graduação Subjetiva de Satisfação de Johnson gradua a satisfação do paciente em: completamente satisfeito; satisfeito com mínimas restrições; satisfeito com maiores restrições e insatisfeito. Os pacientes foram avaliados antes do procedimento e após o período de uma semana, um mês, três meses; seis meses e 12 meses. Foi obtida a devida aprovação da comissão de ética da instituição e não houve conflito de interesses. Utilizamos o teste $t$ de student para variáveis continuas e o teste do Qui-Quadrado para variáveis dicotômicas. Foi adotado como significância estatística um $p<0,05$. Os dados foram analisados usando software estatístico (SPSS 12.0, SPSS Inc., Chicago, IL).

\section{RESULTADOS}

O tempo de seguimento pós-operatório variou de 12 a 26 meses. Quanto ao sexo, doze (85\%) pacientes eram do sexo feminino e cinco (15\%) pacientes do sexo masculino. A idade a média foi de 72,4 anos (variação 61-92 anos). Quanto à raça dez (40\%) pacientes eram brancas, sete (10\%) pardas.

Pela avaliação com a Escala Visual Analógica da dor houve uma queda significativa de 6.4 pontos ao final de doze meses de seguimento em comparação ao período pré-operatório $(p<00,5)$ (Tabela 1).

A avaliação funcional realizada pelo Oswestry Disability Index 2.0 mostrou $88 \%$ de resultados excelentes ou bons, sendo que sete pacientes (41\%) apresentaram excelentes resultados e oito pacientes (47\%) resultados bons. Dois pacientes (12\%) mantiveram sua avaliação funcional inalterada (Figura 1, Tabela 2).

Quanto à graduação subjetiva de satisfação $82 \%$ declararamse satisfeitos, sendo que $59 \%$ estavam completamente satisfeitos e 23\% satisfeitos com mínimas restrições. Três pacientes (18\%) declararam-se insatisfeitos com o resultado do procedimento (Figura 2, Tabela 3)

As complicações encontradas foram: 02 pacientes com extravasamento de cimento para o disco intervertebral e 01 paciente com extravasamento para o canal vertebral, sem repercussão clínica. Um paciente sofreu infarto agudo do miocárdio (IAM) logo após a realização da cimentação da vértebra e teve que ser encaminhado a unidade coronariana. Nenhum paciente referiu piora da dor logo após o procedimento. 
Tabela 1. Escala Visual Analógica - EVA.

\begin{tabular}{c|c|c|c|c|c|c}
\hline Paciente & Pré & $\mathbf{1}$ sem & 1mes & 3 meses & $\mathbf{6}$ meses & 1 ano \\
\hline JBS & 8 & 5 & 1 & 1 & 1 & 1 \\
\hline MLM & 10 & 3 & 0 & 0 & 0 & 0 \\
\hline PPS & 10 & 7 & 7 & 5 & 2 & 2 \\
\hline FRT & 9 & 2 & 5 & 2 & 2 & 2 \\
\hline MAB & 8 & 2 & 2 & 2 & 2 & 2 \\
\hline JAV & 8 & 6 & 5 & 7 & 7 & 7 \\
\hline WHS & 10 & 1 & 1 & 0 & 0 & 0 \\
\hline ECL & 10 & 5 & 5 & 5 & 2 & 2 \\
\hline AGS & 10 & 5 & 5 & 5 & 5 & 5 \\
\hline RRP & 8 & 2 & 2 & 0 & 0 & 0 \\
\hline LSS & 9 & 0 & 0 & 0 & 0 & 0 \\
\hline DFG & 9 & 6 & 5 & 5 & 5 & 5 \\
\hline AAT & 9 & 5 & 5 & 0 & 1 & 0 \\
\hline RDO & 8 & 2 & 2 & 2 & 2 & 2 \\
\hline PMO & 7 & 0 & 2 & 2 & 2 & 2 \\
\hline TRV & 8 & 3 & 3 & 3 & 3 & 3 \\
\hline BCP & 10 & 2 & 0 & 5 & 7 & 7 \\
\hline
\end{tabular}

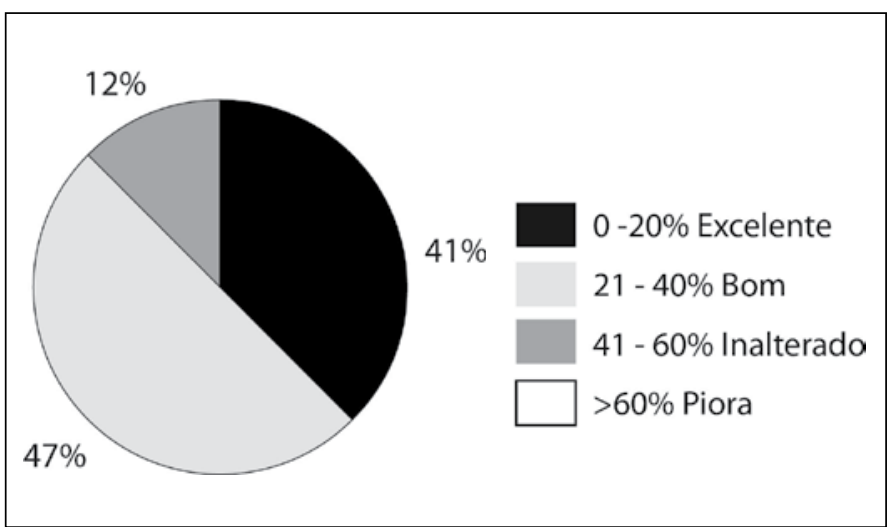

Figura 1. Índice Owestry de Incapacidade.

Tabela 2. Índice Owestry de Incapacidade.

\begin{tabular}{c|c|c|c|c|c|c}
\hline Paciente & Pré & $\mathbf{1 ~ s e m ~}$ & $\mathbf{1 m e s}$ & $\mathbf{3}$ meses & $\mathbf{6}$ meses & $\mathbf{1}$ ano \\
\hline JBS & $58 \%$ & $28 \%$ & $14 \%$ & $18 \%$ & $18 \%$ & $20 \%$ \\
\hline MLM & $65 \%$ & $14 \%$ & $12 \%$ & $18 \%$ & $18 \%$ & $18 \%$ \\
\hline PPS & $85 \%$ & $26 \%$ & $30 \%$ & $22 \%$ & $24 \%$ & $24 \%$ \\
\hline FRT & $45 \%$ & $21 \%$ & $35 \%$ & $18 \%$ & $22 \%$ & $23 \%$ \\
\hline MAB & $58 \%$ & $18 \%$ & $14 \%$ & $16 \%$ & $16 \%$ & $18 \%$ \\
\hline JAV & $66 \%$ & $26 \%$ & $35 \%$ & $35 \%$ & $40 \%$ & $45 \%$ \\
\hline WHS & $64 \%$ & $20 \%$ & $10 \%$ & $12 \%$ & $12 \%$ & $10 \%$ \\
\hline ECL & $82 \%$ & $25 \%$ & $28 \%$ & $34 \%$ & $34 \%$ & $38 \%$ \\
\hline AGS & $52 \%$ & $45 \%$ & $41 \%$ & $30 \%$ & $31 \%$ & $36 \%$ \\
\hline RRP & $44 \%$ & $23 \%$ & $18 \%$ & $18 \%$ & $12 \%$ & $8 \%$ \\
\hline LSS & $68 \%$ & $23 \%$ & $32 \%$ & $26 \%$ & $18 \%$ & $12 \%$ \\
\hline DFG & $81 \%$ & $48 \%$ & $38 \%$ & $26 \%$ & $32 \%$ & $24 \%$ \\
\hline AAT & $80 \%$ & $39 \%$ & $21 \%$ & $20 \%$ & $14 \%$ & $10 \%$ \\
\hline RDO & $74 \%$ & $32 \%$ & $34 \%$ & $24 \%$ & $26 \%$ & $26 \%$ \\
\hline PMO & $43 \%$ & $28 \%$ & $28 \%$ & $31 \%$ & $30 \%$ & $30 \%$ \\
\hline TRV & $52 \%$ & $29 \%$ & $21 \%$ & $21 \%$ & $24 \%$ & $32 \%$ \\
\hline BCP & $82 \%$ & $18 \%$ & $18 \%$ & $30 \%$ & $41 \%$ & $50 \%$ \\
\hline
\end{tabular}

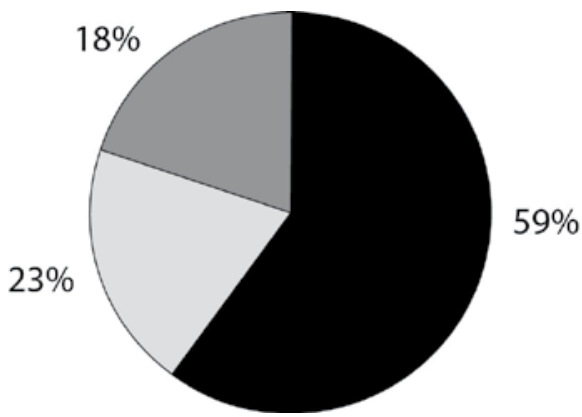

Completamente satisfeito

Satisfeito com mínimas restrições

Satisfeito com maiores restrições

Insatisfeito

Figura 2. Graduação de Satisfação Pessoal de Jhonson et al.

Tabela 3. Graduação Subjetiva de Satisfação de Jhonson.

\begin{tabular}{c|c|c|c|c|c|c}
\hline Paciente & Pré & $\mathbf{1}$ sem & 1mes & 3 meses & $\mathbf{6}$ meses & 1 ano \\
\hline JBS & & 2 & 1 & 1 & 1 & 1 \\
\hline MLM & & 1 & 1 & 1 & 1 & 1 \\
\hline PPS & & 3 & 2 & 2 & 2 & 2 \\
\hline FRT & & 1 & 1 & 1 & 1 & 1 \\
\hline MAB & & 2 & 1 & 2 & 1 & 1 \\
\hline JAV & & 4 & 3 & 2 & 2 & 4 \\
\hline WHS & & 1 & 1 & 1 & 1 & 1 \\
\hline ECL & & 2 & 1 & 1 & 1 & 1 \\
\hline AGS & & 3 & 3 & 2 & 2 & 2 \\
\hline RRP & & 1 & 1 & 1 & 1 & 4 \\
\hline LSS & & 1 & 1 & 1 & 1 & 4 \\
\hline DFG & & 2 & 2 & 2 & 2 & 2 \\
\hline AAT & & 2 & 1 & 1 & 1 & 1 \\
\hline RDO & & 2 & 2 & 1 & 1 & 1 \\
\hline PMO & & 1 & 2 & 2 & 2 & 2 \\
\hline TRV & & 1 & 1 & 1 & 1 & 1 \\
\hline
\end{tabular}

\section{DISCUSSÃO}

Atualmente, desfechos como qualidade de vida relacionada à saúde, capacidade funcional, escalas de dor e satisfação têm sido enfatizados, por possibilitarem a análise da situação de saúde e as manifestações da doença na vida do indivíduo em sua própria perspectiva (subjetividade), complementando os dados clínicos objetivos e possibilitando a incorporação da opinião dos pacientes na tomada de decisão.

Muitos pacientes melhoram com o tratamento conservador que inclui repouso e analgésicos para liberação de dor, além do tratamento de base para a osteoporose. Para aqueles pacientes nos quais o tratamento conservador não tenha sido efetivo, estaria indicado a cimentação óssea do corpo vertebral fraturado. A literatura atual é controversa quanto ao momento ideal para realização do procedimento. Há autores que defendem tempo de espera mínimo de três meses para indicar o procedimento; em contrapartida trabaIho recente ${ }^{17}$ sugere a realização precoce (aguda) da cimentação em favor de melhores resultados e menores taxas de complicação. Em nosso serviço acreditamos que o momento ideal deva ser in- 
dividualizado através de um acompanhamento rigoroso, onde a melhora gradual possa ser levada em consideração para a tomada de decisão. Para o presente estudo utilizamos o tempo de oito semanas como limite para definir falha terapêutica do tratamento conservador levando em consideração dados da literatura atual|-6.

Em nossa casuística houve redução significativa da dor (EVA) e da incapacidade funcional (Owestry), com resultado mantido por todo o segmento na maioria dos pacientes, resultado esse compartilhado pela literatura médica internacional ${ }^{4-6,17}$ e, também em nosso meio ${ }^{18,19}$

As complicações apresentadas em nossa série (extravasamento assintomático de cimento e efeitos cardiovasculares do polimetil- metacrilato) estão bem documentados na literatura. Embora não tenhamos apresentado nenhum caso de fratura do nível adjacente, acreditamos que isso se deva ao pequeno tamanho da amostra e curto tempo de segmento.

\section{CONCLUSÃO}

A cifoplastia mostrou-se efetiva em melhorar a função e liberar a dor em pacientes com fratura vertebral por insuficiência.

A ocorrência de uma complicação grave (IAM) chama atenção para necessidade de suporte hospitalar adequado durante a realização do procedimento.

\section{REFERÊNCIAS}

1. NIH Consensus Development Panel on Osteoporosis Prevention, Diagnosis, and Therapy, March 7-29, 2000: highlights of the conference. South Med J. 2001;94(6):569-73.

2. Silverman SL. The clinical consequences of vertebral compression fracture. Bone. 1992;13(Suppl.2):S27-31.

3. Cooper C, Atkinson EJ, O'Fallon WM, Melton LJ 3rd. Incidence of clinically diagnosed vertebral fractures: a population-based study in Rochester, Minnesota, 1985-1989. J Bone Miner Res. 1992;7(2):221-7.

4. Coumans JV, Reinhardt MK, Lieberman IH. Kyphoplasty for vertebral compression fractures: 1-year clinical outcomes from a prospective study. J Neurosurg. 2003;99(1 Suppl):44-50.

5. Lyles KW, Gold DT, Shipp KM, Pieper CF, Martinez S, Mulhausen PL. Association of osteoporotic vertebral compression fractures with impaired functional status. Am J Med. 1993;94(6):595-601

6. Evans AJ, Jensen ME, Kip KE, DeNardo AJ, Lawler GJ, Negin GA, et al. Vertebral compression fractures: pain reduction and improvement in functional mobility after percutaneous polymethylmethacrylate vertebroplasty retrospective report of 245 cases. Radiology. 2003;226(2):366-72.

7. Kado DM, Duong T, Stone KL, Ensrud KE, Nevitt MC, Greendale GA, et al. Incident vertebral fractures and mortality in older women: a prospective study. Osteoporos Int. 2003;14(7):589-94.

8. Kramer DL, Rodgers WB, Mansfield FL. Transpedicular instrumentation and short-segment fusion of thoracolumbar fractures: a prospective study using a single instrumentation system. J Orthop Trauma. 1995;9(6):499-506.

9. Benson DR, Burkus JK, Montesano PX, Sutherland TB, McLain RF. Unstable thoracolumbar and lumbar burst fractures treated with the AO fixateur interne. J Spinal Disord. 1992;5(3):335-43.
10. McLain RF, Sparling E, Benson DR. Early failure of short-segment pedicle instrumentation for thoracolumbar fractures. A preliminary report. J Bone Joint Surg Am. 1993;75(2):162-7.

11. Esses SI, Botsford DJ, Wright T, Bednar D, Bailey S. Operative treatment of spinal fractures with the AO internal fixator. Spine (Phila Pa 1976). 1991;16(3 Suppl):S146-50.

12. Kaneda K, Taneichi H, Abumi K, Hashimoto T, Satoh S, Fujiya M. Anterior decompression and stabilization with the Kaneda device for thoracolumbar burst fractures associated with neurological deficits. J Bone Joint Surg Am. 1997;79(1):69-83.

13. Korovessis P, Baikousis A, Zacharatos S, Petsinis G, Koureas G, lliopoulos P. Combined anterior plus posterior stabilization versus posterior short-segment instrumentation and fusion for mid-lumbar (L2-L4) burst fractures. Spine (Phila Pa 1976). 2006:31(8):859-68.

14. McDonough PW, Davis R, Tribus C, Zdeblick TA. The management of acute thoracolumbar burst fractures with anterior corpectomy and Z-plate fixation. Spine (Phila Pa 1976). 2004;29(17):1901-8.

15. Arrington ED, Smith WJ, Chambers HG, Bucknell AL, Davino NA. Complications of iliac crest bone graft harvesting. Clin Orthop Relat Res. 1996;(329):300-9.

16. Korovessis P, Baikousis A, Zacharatos S, Petsinis G, Koureas G, Iliopoulos P. Combined anterior plus posterior stabilization versus posterior short-segment instrumentation and fusion for mid-lumbar (L2-L4) burst fractures. Spine (Phila Pa 1976). 2006;31(8):859-68.

17. Klazen CA, Lohle PN, de Vries J, Jansen FH, Tielbeek AV, Blonk MC, et al. Vertebroplasty versus conservative treatment in acute osteoporotic vertebral compression fractures (Vertos II): an open-label randomised trial. Lancet. 2010;376(9746):1085-92.

18. Pinto R, Matos R, Neves N, Tulha JM, Cabral AT, Almeida L. A vertebroplastia no tratamento das fraturas vertebrais osteoporóticas dolorosas. Coluna/Columna. 2007:6(3):136-40.

19. Oliveira FM, Rodrigues AG, Bastos Junior JOC, Yamazoto C, Kusabara R. Resultados clínicos da vertebroplastia em pacientes com fratura da coluna vertebral por osteoporose. Coluna/ Columna. 2007;6(1):28-33. 